Bryn Mawr College

Scholarship, Research, and Creative Work at Bryn Mawr

College

Physics Faculty Research and Scholarship

Physics

1998

\title{
Phase and Risetime Dependence Using RF Pulses in Multiphoton Processes
}

W.M. Griffith

Michael W. Noel

Bryn Mawr College, mnoel@brynmawr.edu

T. F. Gallagher

Let us know how access to this document benefits you.

Follow this and additional works at: http://repository.brynmawr.edu/physics_pubs

Part of the Physics Commons

\section{Custom Citation}

W. M. Griffith, Michael W. Noel, and T. F. Gallagher, "Phase and Risetime Dependence Using RF Pulses in Multiphoton Processes," Phys. Rev. A 57, 3698 (1998).

This paper is posted at Scholarship, Research, and Creative Work at Bryn Mawr College. http://repository.brynmawr.edu/physics_pubs/74

For more information, please contact repository@brynmawr.edu. 


\title{
Phase and rise-time dependence using rf pulses in multiphoton processes
}

\author{
W. M. Griffith, Michael W. Noel, and T. F. Gallagher \\ Department of Physics, University of Virginia, Charlottesville, Virginia 22901
}

(Received 12 May 1997; revised manuscript received 26 November 1997)

\begin{abstract}
With this experiment we demonstrate that excitation of a two-state system with radio-frequency fields differing in phase by $90^{\circ}$ produces nonintuitively different results, even for very long pulses. In addition, we show how the phase dependence of the transition probability of long pulses can be easily understood by using the single cycle time propagator. Finally, we have found surprising results for real pulses in the strong-field regime, i.e., pulses having appreciable rise and fall times. [S1050-2947(98)01104-4]
\end{abstract}

PACS number(s): $32.80 . \mathrm{Wr}$

\section{INTRODUCTION}

The physics of a strong, sinusoidally oscillating laser field interacting with an atom has been largely understood in terms of Floquet theory [1], which takes account of the strong, and hence nonperturbative, field effects and assumes a constant field amplitude. Floquet theory is often done by using the well-defined periodicity of the interaction Hamiltonian and transforming it into the time-independent Floquet Hamiltonian, which is represented by an infinite matrix in the basis of states "dressed", with $n$ photons, where $n=0, \pm 1, \pm 2, \ldots$, or more simply dressed states. By reextending the Floquet picture into the time domain, it has also been shown to describe sinusoidally oscillating fields that are slowly varying in either amplitude [2-7] or frequency [810]. Other studies of these systems, following an alternative route of investigation, have deepened our understanding of the effects of finite duration pulses [11-14]. These descriptions exploit the periodic nature of the exciting field in a different way by building up the response of the atom one cycle at a time. While many of the underlying concepts that connect the latter approach to Floquet theory have been exposed, it is unclear how both of these paths to understanding a generalized excitation may be applied to real pulses, such as those produced by a short-pulse laser that contains only a few optical cycles. Real pulse characteristics such as phase, finite pulse length, and envelope shape affect the response of the system and as such are a necessary part of any description of an arbitrary excitation. In particular, when the envelope shape changes appreciably on a time scale comparable to the period of the carrier, it is clear that the correspondence between Floquet theory and experimental results must break down.

In our experimental and theoretical studies, we are now able to decompose the separate effects of pulse length, phase, and envelope rise time from the well-known time and phase averaged response [1]. We present here the observation of the response of a two-level system to radio-frequency (rf) pulses in which we can precisely control each of the parameters mentioned above. First, we study the near ideal cases, examining how the transition probability of the two-level system differs for an rf pulse with a sine or a cosine phase. We additionally examine how this response to a pulse of many cycles at a fixed phase can be built up from the response to a single cycle of the same phase. Second, we ex- amine how departures from the ideal rf pulse, either in phase or by the imposition of a finite envelope rise time, can be related to the simpler ideal case.

\section{THEORY}

\section{A. Single cycle Floquet approach}

If a system consisting of $M$ states is described at $t=0$ by the state vector $\mathbf{v}_{\mathbf{0}}$, at some later time $t$ it is described by the vector $\mathbf{v}(t)$ related to $\mathbf{v}_{\mathbf{0}}$ by the unitary transformation matrix $U(t)$. Explicitly,

$$
\mathbf{v}(t)=U(t) \mathbf{v}_{\mathbf{0}}
$$

If the $M$ basis functions used to describe the system are energy eigenfunctions and there is no time-dependent coupling between them, $U(t)$ is a diagonal matrix with elements $U_{j j}=e^{-i W_{j} t}$, where $W_{j}$ is the energy of the $j$ th eigenstate. The effect of the time evolution is simply to alter the phase of the $j$ th state by $-W_{j} t$. Explicitly,

$$
\mathbf{v}_{j}(t)=e^{-i W_{j} t} \mathbf{v}_{\mathbf{0} j}
$$

Now imagine that we introduce a periodic interaction, of period $\tau$, which couples the energy eigenstates. Over one cycle of the interaction the evolution is described by the unitary transformation $U$ and the resulting state vector after one period $\mathbf{v}_{\mathbf{1}} \equiv \mathbf{v}(\tau)$ is related to the original state vector by

$$
\mathbf{v}_{\mathbf{1}}=U \mathbf{v}_{\mathbf{0}}
$$

We can diagonalize $U$ with a unitary matrix $T$ to obtain $U^{\prime}$, with diagonal elements $U_{j j}^{\prime}=e^{i \phi_{j}}$ and where $U=T^{-1} U^{\prime} T$. This relation allows us to transform Eq. (3) into the basis defined by $T$,

$$
\mathbf{v}_{\mathbf{1}}^{\prime}=U^{\prime} \mathbf{v}_{\mathbf{0}}^{\prime}
$$

where we have defined $\mathbf{v}_{\mathbf{1}}^{\prime} \equiv T \mathbf{v}_{\mathbf{1}}$ and $\mathbf{v}_{\mathbf{0}}^{\prime} \equiv T \mathbf{v}_{\mathbf{0}}$. If there are $N$ periods of the interaction, the time evolution matrix is $U^{N}$ and after $N$ periods the system is described by the state vector $\mathbf{v}_{N} \equiv \mathbf{v}(N \tau)$ given by

$$
\mathbf{v}_{N}=U^{N} \mathbf{v}_{\mathbf{0}}
$$


Defining $\mathbf{v}_{N}^{\prime} \equiv T \mathbf{v}_{N}$ and using the fact that $U^{N}=T^{-1}\left(U^{\prime}\right)^{N} T$, we can follow the same reasoning as in Eq. (4) and arrive at

$$
\mathbf{v}_{N}^{\prime}=\left(U^{\prime}\right)^{N} \mathbf{v}_{\mathbf{0}}^{\prime}
$$

Since $\left(U^{\prime}\right)^{N}$ is diagonal with elements $e^{i N \phi_{j}}$, the effect of $N$ cycles is to alter the phase of the $j$ th component by $N \phi_{j}$, so that

$$
\mathbf{v}_{N j}^{\prime}=e^{i N \phi_{j}} \mathbf{v}_{\mathbf{0} j}^{\prime}
$$

This is precisely the same effect as the evolution of Eq. (1) for energy eigenstates, as shown by Eq. (2), so we identify the rate of phase accumulation of state $j$ as its Floquet energy (also called the quasienergy). Explicitly,

$$
W_{j}=\frac{-\phi_{j}}{\tau}
$$

and the Floquet eigenstates are related to the energy eigenstates by the unitary transformation $T$, which does not depend in any way on the number of periods. Note that the eigenvalues of $U$ that define the $\phi_{j}$ allow for the substitution $\phi_{j} \rightarrow \phi_{j}-2 \pi n$, where $n=0, \pm 1, \ldots$, which allow an arbitrariness in the Floquet energies $W_{j} \rightarrow W_{j}+n \omega$, where $\omega=2 \pi / \tau$.

In practice, the matrix $U$ can be determined by integrating the Schrodinger equation over one period of the interaction using the energy eigenstates. Simply finding the eigenvalues of $U$ gives the elements of the diagonal matrix $U^{\prime}$ and hence the Floquet energy eigenvalues. Finding the unitary transformation $T$ that connects $U$ and $U^{\prime}$, lets us describe the Floquet eigenvector $\mathbf{v}_{\mathbf{0}}^{\prime}$ in terms of the energy eigenvector.

\section{B. Transitions in a two-level system}

We assume that initially all population is in one of the two energy eigenstates, state 1, so that in the basis of energy eigenstates

$$
\mathbf{v}_{\mathbf{0}}=\left(\begin{array}{l}
1 \\
0
\end{array}\right)
$$

After $N$ cycles the state vector is given by

$$
\mathbf{v}_{N}=U^{N} \mathbf{v}_{\mathbf{0}}
$$

where $U$ is the single cycle evolution matrix, which can be diagonalized to obtain $U^{\prime}$. We can express $U^{N}$ of Eq. (10) as

$$
U^{N}=T^{-1}\left(\begin{array}{cc}
e^{i N \phi_{1}} & 0 \\
0 & e^{i N \phi_{2}}
\end{array}\right) T .
$$

The transition probability to state 2 is then given by

$$
\left|\mathbf{v}_{N 2}\right|^{2}=4\left|T_{12} T_{11}\right|^{2} \sin ^{2}\left(\frac{\Phi}{2}\right),
$$

where the phase difference $\Phi \equiv N\left(\phi_{1}-\phi_{2}\right)$. The transition probability depends on the single cycle transformation matrix $T$ and an oscillatory term that represents Rabi oscillations. Note that it is simply the product of the difference of the Floquet energies and the time. For some purposes it is

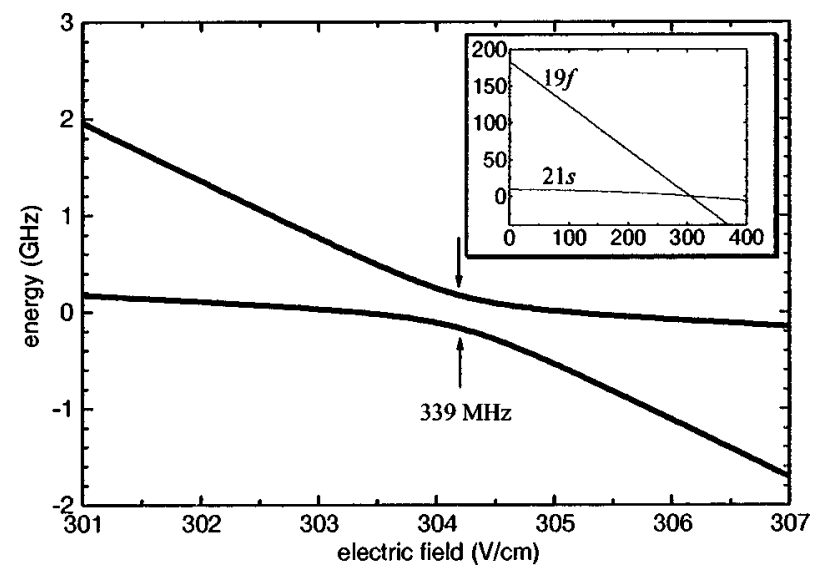

FIG. 1. Energy-level diagram showing the K states adiabatically connected to the $21 \mathrm{~s}$ and $19 f$ states vs static field. We define the static field in the figure such that the point where $E=0$ corresponds to the central point of the anticrossing. All data described in this article were taken at this static field. The inset shows the energy levels of the two states vs the true electric field. The actual location of the crossing point is $304.2 \mathrm{~V} / \mathrm{cm}$.

useful to average over the Rabi oscillations, i.e., take the average over $\Phi$. Doing so yields

$$
\overline{\left|\mathbf{v}_{N 2}\right|^{2}}=2\left|T_{12} T_{11}\right|^{2}
$$

which depends only on the single-cycle transformation $T$.

The unitary evolution matrix $U$ describing the evolution of the eigenstates over one period can be written as

$$
U=e^{i \Gamma}\left(\begin{array}{cc}
\cos \theta e^{i \delta} & \sin \theta e^{i \phi} \\
-\sin \theta e^{-i \phi} & \cos \theta e^{-i \delta}
\end{array}\right)
$$

The eigenvalues of $U$ are

$$
\lambda_{1,2}=e^{i(\Gamma \pm A)}
$$

where $\cos A \equiv \cos \theta \cos \delta$. Consequently, the Floquet energy eigenvalues differ by $2 A / \tau$ and the phase difference $\Phi=2 N A$. If we take a unitary form for $T$, we are able to calculate the parts of the matrix that we need for the transition probabilities of Eqs. (12) and (13) by diagonalizing $U$. This exercise yields

$$
\left|T_{12} T_{11}\right|^{2}=\frac{1}{4\left(\cot ^{2} \theta \sin ^{2} \delta+1\right)} .
$$

\section{Specific example of a two-level system with a sinusoidal interaction}

Our two-level system, shown in Fig. 1, is found at the first anticrossing of the potassium $21 s-19 f$ states, where we use the convention of labeling our states by their adiabatically connected atomic states at zero electric field. These states, which have been extensively studied $[15,16]$, repel due to the electron core coupling and the center of this anticrossing serves as an ideal location for an experiment for at the avoided crossing the system closely approximates an atom that is at zero field and excited by a laser. In the vicinity of 


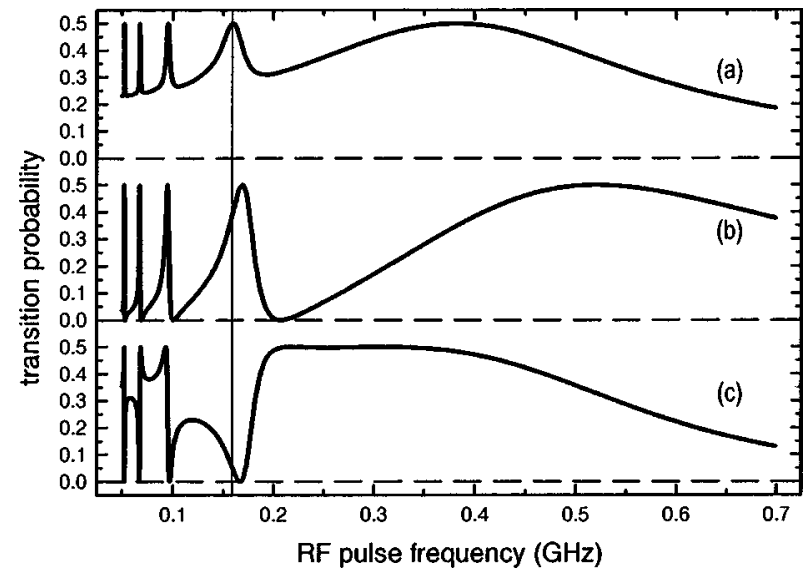

FIG. 2. rf pulse frequency scan with (a) time and phase averaging, (b) sine phase $\left(\phi=90^{\circ}\right)$, and (c) cosine phase $\left(\phi=0^{\circ}\right)$, calculated at an $\mathrm{rf}$ pulse amplitude of $0.87 \mathrm{mV} / \mathrm{cm}$. The thin vertical line is drawn at $160 \mathrm{MHz}$, the location of the three-photon resonance.

the anticrossing, the $21 s$ and $19 f$ states, the latter of which has a linear Stark shift, can be described by the Hamiltonian

$$
H=\left(\begin{array}{cc}
-k E & \frac{\omega_{0}}{2} \\
\frac{\omega_{0}}{2} & 0
\end{array}\right) \text {, }
$$

where $k=546 \mathrm{MHz} /(\mathrm{V} / \mathrm{cm})$ is the slope of state 1 and $\omega_{0} / 2$ is the core coupling so that $\omega_{0}=339 \mathrm{MHz}$ is the minimum energy spacing at the avoided crossing. We have defined the electric field $E$ such that $E=0$ corresponds to the intersection, in the absence of coupling, between the two states. This is the standard description of two levels that cross and have a finite interaction. When transformed to the "upper' and "lower' state basis, which are stationary when $E=0$,

$$
H^{\prime}=\left(\begin{array}{cc}
-\frac{\omega_{0}}{2} & -\frac{k E}{2} \\
-\frac{k E}{2} & \frac{\omega_{0}}{2}
\end{array}\right),
$$

after shifting the zero of energy. This Hamiltonian is the standard description of two levels, separated by $\omega_{0}$, with an interaction that varies with the electric field.

The result of a time-averaged and phase-averaged sinusoidally oscillating electric field $E(t)=E_{\mathrm{rf}} \cos (\omega t+\phi)$ in the interaction described by Eq. (18) was presented by Shirley in 1965 [1]. In the last figure of his paper, Shirley plots the transition probability $P$ as a function of the frequency of the exciting field, obtained from the Floquet energies $W$ using the formula

$$
P=\frac{1}{2}\left[1-4\left(\frac{\partial W}{\partial \omega_{0}}\right)^{2}\right]
$$

In Fig. 2(a) we show the transition probability as a function of frequency calculated using Eq. (19) for our problem with a resonance frequency of $339 \mathrm{MHz}$ and a field amplitude
$E=0.87 \mathrm{~V} / \mathrm{cm}$. We also show in Figs. 2(b) and 2(c) the results obtained by applying the analysis of Sec. II B to the Hamiltonian of Eq. (18) for the two phases $\phi=0$ and $\phi=\pi / 2$. First, it should be noted that the quasienergies used to obtain Fig. 2(a) were produced not by use of the Floquet Hamiltonian, but rather by use of the single cycle propagator, as outlined in Sec. II A. The eigenvalues of the propagator matrix $U$ give the Floquet energies, which through Eq. (19) give time- and phase-averaged transition probabilities. Since it is clear that neither the Floquet energies nor the time- and phase-averaged transition probabilities can depend on $\phi$, the phase of the sinusoid used to generate $U$, it must be true that for every $U=U(\phi)$,

$$
U(\phi)=T^{-1}(\phi) U^{\prime} T(\phi),
$$

where $U^{\prime}$ is independent of $\phi$. Clearly the fixed-phase, timeaveraged, single cycle results obtained using Eq. (13), shown in Figs. 2(b) and 2(c) for a sine $(\phi=\pi / 2)$ and for a cosine $(\phi=0)$, respectively, are quite different from the phaseaveraged result of Fig. 2(a). The surprising result is that a pulse of an integral number of cycles, no matter how many, will follow the envelope defined by the single cycle propagator of the same phase. For example, an integral number of cycles of the sine phase at a frequency just above a multiphoton resonance will transfer no population into the upper state. It has not escaped our attention that the sine and cosine results resemble Fano profiles. The zeros in the transition probabilities, seen near the multiphoton resonances, appear to be a result of interference between the multiphoton resonance and the broad one-photon resonance background. The zeros are always on the high-frequency side of the multiphoton resonances for both the sine and cosine phases.

Unfortunately, the creation of rf pulses with a fixed number of cycles while scanning the rf frequency is difficult to implement experimentally. A different method that exhibits the same effect is to create a single pulse of fixed frequency and number of cycles and scan its amplitude. The frequency of the pulse is chosen such that at some power level in the scan the system will be brought into multiphoton resonance by the ac Stark shift. We have found it convenient to study the three-photon resonance that occurs at $113 \mathrm{MHz}$ at zero rf power. Note that since the levels are pushed further apart with higher power, the low-power side of the resonance is also the side where the rf frequency is larger than necessary for resonance and vice versa. As an example, the results of a calculation made in this fashion of the standard time- and phase-averaged Floquet picture is shown in Fig. 3(a). The rf frequency is $160 \mathrm{MHz}$ and at an amplitude of $\sim 0.9 \mathrm{~V} / \mathrm{cm}$ the three-photon transition is shifted into resonance. The fixedphase, time-averaged results for a sine phase [Fig. 3(b)] and a cosine phase [Fig. 3(c)] differ markedly from the phaseaveraged results in the same fashion as the frequency plots shown above.

\section{EXPERIMENTAL APPROACH}

The experimental setup used here is very similar to what has been described previously [14]. As illustrated in Fig. 4, $\mathrm{K}$ atoms effusing from a resistively heated oven pass between the two plates of a brass transmission line and are 


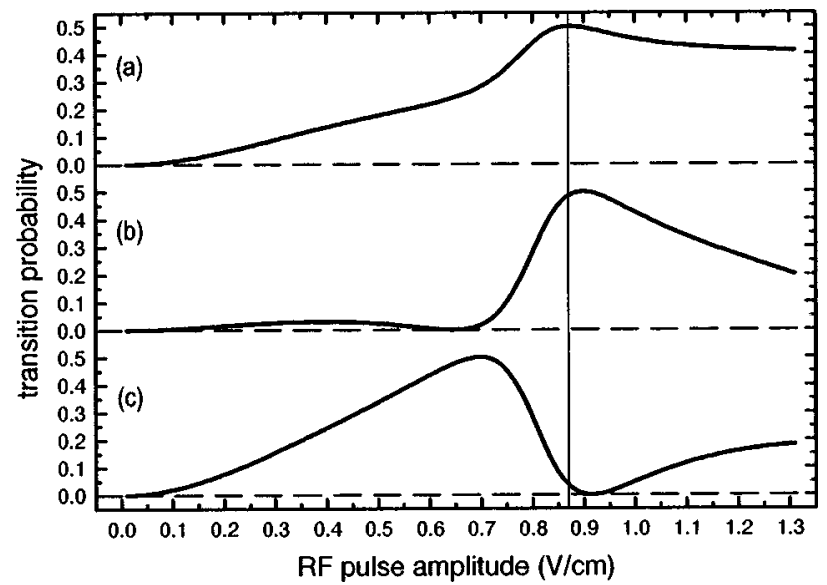

FIG. 3. rf pulse amplitude scan with (a) time and phase averaging, (b) sine phase, and (c) cosine phase, calculated at an rf pulse frequency of $160 \mathrm{MHz}$. The thin vertical line is drawn at 0.87 $\mathrm{V} / \mathrm{cm}$, the location of the three photon resonance.

excited by two counterpropagating nanosecond dye lasers that stimulate the $4 s \rightarrow 4 p$ and $4 p \rightarrow 21 s$ transitions. As shown by the timing diagram in Fig. 5, the atoms are excited in a static field $5 \mathrm{~V} / \mathrm{cm}$ below the avoided crossing. The field is ramped up to the field of the avoided crossing in $300 \mathrm{~ns}$, bringing the atoms adiabatically from the $21 s$ state to the lower state of the avoided crossing. There the desired rf pulse is applied to drive transitions to the upper state. After the rf field is turned off, the static field is reduced in $300 \mathrm{~ns}$ to $5 \mathrm{~V} / \mathrm{cm}$ below the avoided crossing, so those atoms that are in the lower (upper) state pass adiabatically to the $21 \mathrm{~s}$ $(19 f)$ state. Finally, the field ionization pulse with an amplitude of $2400 \mathrm{~V} / \mathrm{cm}$ is applied, which is sufficient to ionize the $19 f$ but not the $21 s$ state. The resulting ions pass through a hole in the top plate of the transmission line and are detected by a dual microchannel plate detector, whose amplified signal is recorded with a gated integrator and stored on a personal computer (PC).

The transmission line's geometry is such that it has a characteristic impedance of $50 \Omega$ over a broad frequency range. The lower plate is connected to the outer conductor of the BNC connectors at each end by 100-pF capacitors. These capacitors have negligible impedance at high $(>100 \mathrm{MHz})$ frequencies, but high impedance at low $(<1 \mathrm{MHz})$ frequencies, allowing us to apply the static field and the slowly rising field ionization pulse to the lower plate without disturbing the rf field, which is traveling down the transmission line. The upper plate is connected to the center pin of the

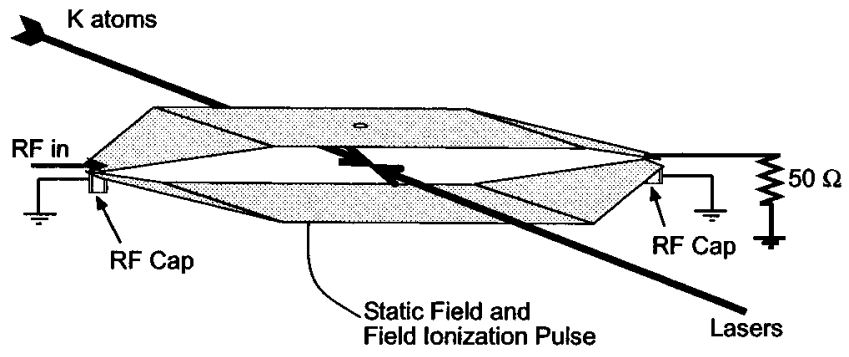

FIG. 4. Diagram of our experimental setup.

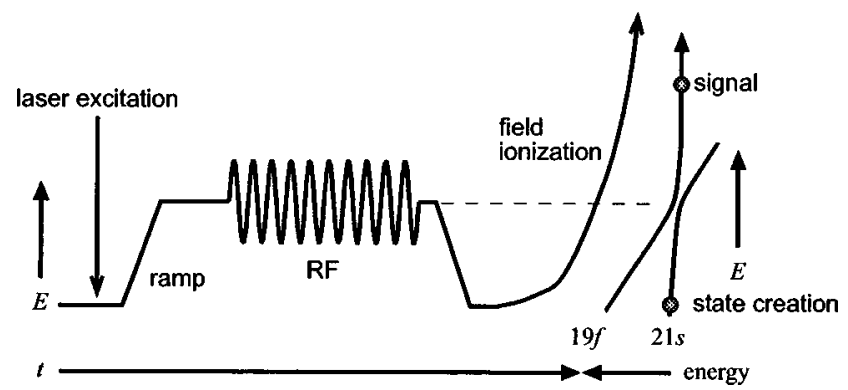

FIG. 5. Electric field experienced by the $\mathrm{K}$ atoms as a function of time in our experiment.

BNC connectors, so that negative voltages (with respect to the outer conductor on the BNC connection) cause an increase in the electric field between the plates.

The phase-locked rf pulses are created in a fashion slightly different from our previous experiment [14]. Instead of using a FM feedback mechanism, we use a Tektronix AWG2040 arbitrary waveform generator (AWG) that can generate a very long sinusoidal pulse along with a square pulse that is locked to its phase. The square pulse from the AWG is used to trigger a Philips PM5785B pulse generator [or a Hewlett-Packard (HP) 8082A pulse generator for the variable rise-time pulses], whose output is a pulse with a variable delay and width. The rf output from the AWG is mixed with the pulse out of the Philips (or HP) pulse generator in a pair of cascaded Watkins Johnson M1A mixers. By mixing the rf and the pulse in this way we create an $\mathrm{rf}$ pulse whose phase and length can be precisely adjusted by changing the delay and length of the pulse from the Philips pulse generator. The resultant rf pulse is then amplified by a Mini-Circuits Laboratories ZHL-42W amplifier, attenuated by a variable amount using a Watkins Johnson WJ-G1 voltage controlled attenuator governed by the PC, and then further amplified by an Amplifier Research 1W1000 amplifier. The fully amplified output is combined with a voltage ramp supplied by a HP $8112 \mathrm{~A}$ pulse generator. The $\mathrm{rf}+$ ramp pulse combination is fed into the vacuum chamber and applied to one end of the transmission line. During the rf pulse, the ramp pulse remains flat to within $\sim 20 \mathrm{mV} / \mathrm{cm}$. The other end of the transmission line is coupled out of the chamber and terminated in $50 \Omega$ on a Tektronix TDS 520B digital oscilloscope, where we can also monitor and store the actual rf pulse shape, which can then be downloaded to the PC. The simplicity of this setup, compared to the FM feedback methods of phase locking, allows us to make rf pulses of definite phase and lengths exceeding 120 cycles that remain stable for extended periods of time.

\section{RESULTS AND DISCUSSION}

The experimental response of $\mathrm{K}$ atoms prepared in the lower state of our chosen anticrossing has a remarkable dependence on pulse phase, as shown in Fig. 6. Here the pulse length was 30 cycles at a frequency of $160 \mathrm{MHz}$ and the rf pulse phase seen by the atoms is the $\phi=90^{\circ}$ sine phase [Fig. 6(a)] and the $\phi=0^{\circ}$ cosine phase [Fig. 6(b)]. The figure insets show the rf pulse observed on the oscilloscope after passing through the transmission line, which were determined to be $\sim 30^{\circ}$ shifted from the phase seen by the atoms 


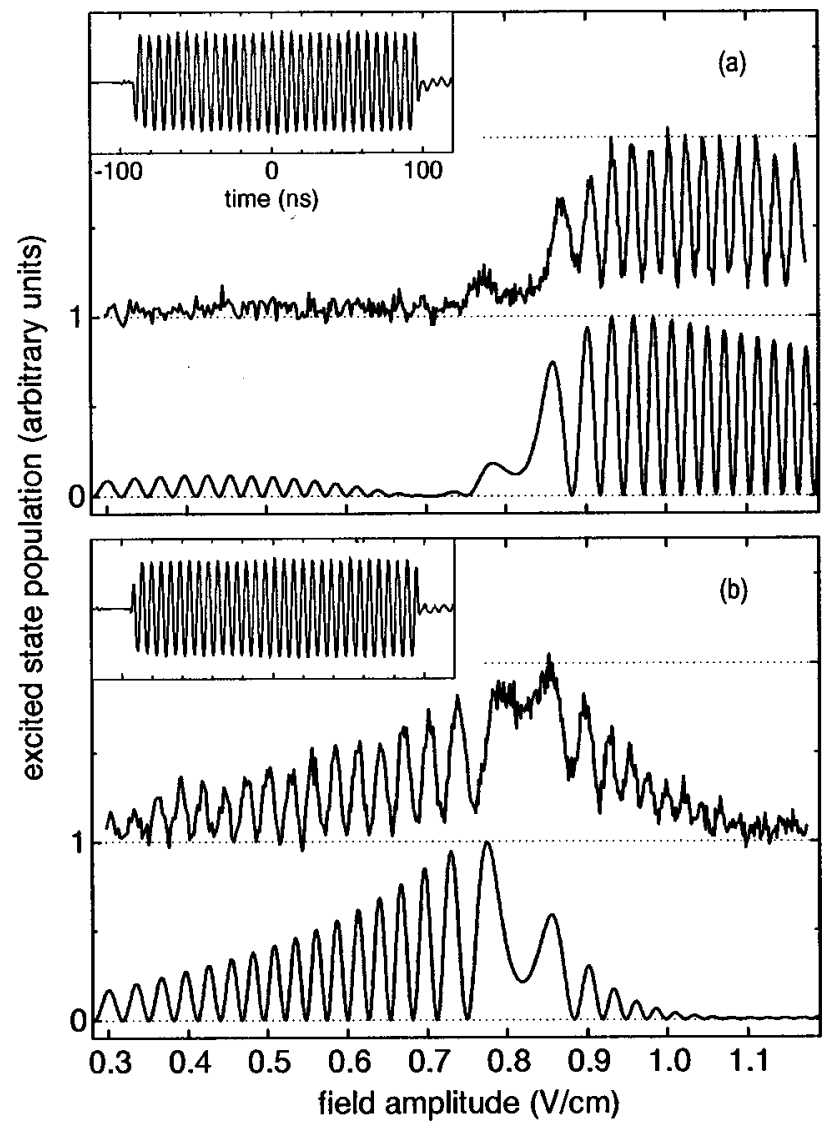

FIG. 6. Experimental result of amplitude scans with 30 cycles at a frequency of $160 \mathrm{MHz}$, where the dependence on rf pulse phase is (a) $\phi=90^{\circ}$, or sine phase, (b) $\phi=0^{\circ}$, or cosine phase. The data in the main graphs were taken with the corresponding rf pulse shown in its inset, which was recorded as discussed in the text. The observed rf pulses are shifted from this phase by $30^{\circ}$. The simulations shown immediately below the data were calculated using an rf pulse of 30 cycles and a 1.5-ns rise time.

by comparison with numerical simulations. The envelope of the transition probability in Fig. 6(a) for an rf pulse excitation with a sine phase matches the expected result shown in Fig. 3(b). The data in Fig. 6(b), where the rf pulse has a cosine phase, have an envelope that also compares well with the prediction shown in Fig. 3(c). Slight disparities in the high-amplitude region can be attributed to the finite rise time of the rf pulse. The result of direct integration of the Schrödinger equation for both of these cases appear immediately below the data. In these calculations, we tried to mimic as closely as possible the actual rf pulse shape experienced by the atoms, including the precise number of cycles and the pulse rise time.

The experimental results for the sine phase rf pulse excitations of different pulse lengths are shown in Fig. 7, for pulse lengths of $1,3,12$, and 90 cycles at $160 \mathrm{MHz}$. As explained previously [14], the transition probabilities for the many cycle pulses can be explained in terms of the single cycle response of the same phase. For example, one common aspect in all of the data for the multiple cycle rf pulses is the very low transition probability in the low-amplitude region $(<0.8 \mathrm{~V} / \mathrm{cm})$ and is a consequence of the fact that the onecycle pulse also has a very low transition probability there. The only significant difference between the data for different

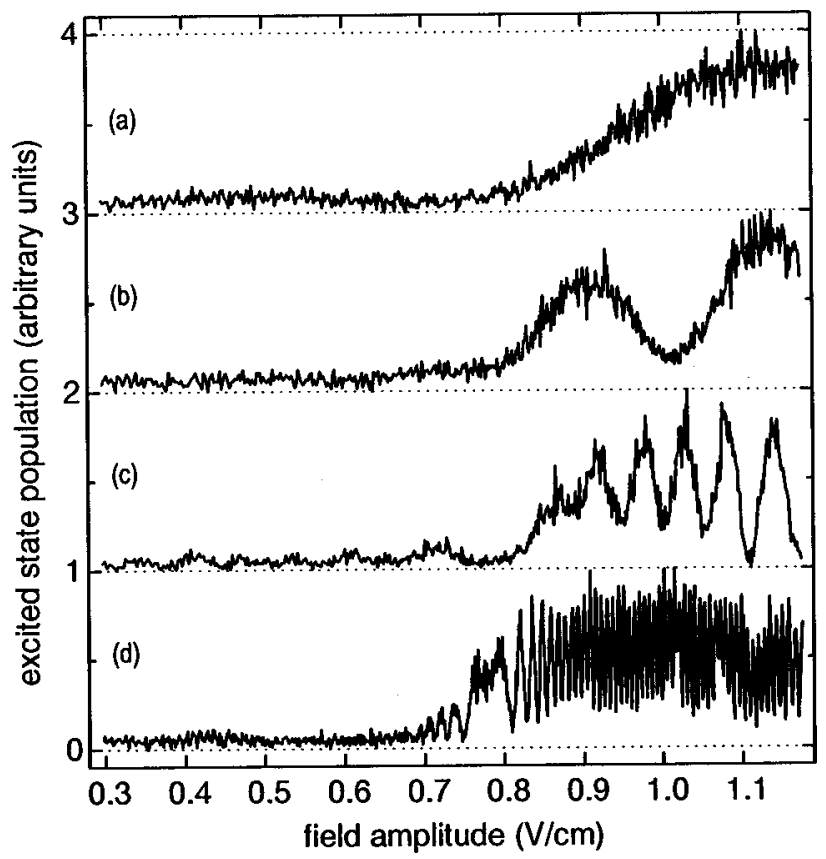

FIG. 7. Experimental result of amplitude scans for the rf pulse sine phase, showing the dependence on the pulse length: (a) 1 cycle, (b) 3 cycles, (c) 12 cycles, and (d) 90 cycles.

pulse lengths is in the number of Rabi oscillations per unit of rf pulse amplitude.

The data already presented were taken under the nearly ideal rf pulse conditions. A closer study of the dependence of the transition probability on rf pulse phase was done with pulse lengths of ten cycles at $160 \mathrm{MHz}$, as shown in Fig. 8, for (a) $\phi=90^{\circ}$, (b) $\phi=60^{\circ}$, (c) $\phi=0^{\circ}$, and (d) $\phi=-30^{\circ}$. The inset oscilloscope traces again represent the $\mathrm{rf}$ pulse used (recall the $\sim 30^{\circ}$ phase shift from the actual rf pulse phase) in each data set. It can be observed from these data that the amplitude scan can be divided into two regions separated by the three-photon resonance at $0.87 \mathrm{~V} / \mathrm{cm}$. The low-amplitude region $(<0.8 \mathrm{~V} / \mathrm{cm})$ is almost completely quiescent for the $\phi=90^{\circ}$ sine phase and rises slightly as the phase changes to $60^{\circ}$. The high-amplitude region $(>0.9 \mathrm{~V} / \mathrm{cm})$ is most suppressed for the $\phi=0^{\circ}$ cosine phase and rises rapidly as the phase changes to $-30^{\circ}$. At what appears to be the most nonideal rf pulse phase presented here, at $\phi=-30^{\circ}$, it is seen that neither the low- nor the high-amplitude regions are suppressed and transitions can be seen for nearly any amplitude.

Next we study the effect of excitation using an rf pulse with an appreciable rise time (we always use equal rise and fall times). As shown in Fig. 9, a rise time of only one cycle leaves the $\phi=90^{\circ}$ sine phase rf pulse results almost completely unchanged from the short-rise-time case. In the $\phi=0^{\circ}$ cosine phase rf pulse there is a suppression of the oscillations in the low-amplitude region and an increased response in the high-amplitude region compared to the shortrise-time case. The difficulty of determining the precise rf pulse phases shown in the insets of Fig. 9 has diminished importance here because of the fact that numerical studies show that a rise time of this magnitude produces a response that is nearly independent of phase. Surprisingly, both responses are almost identical to the response to a fast-risetime sine phase rf pulse, but are quite dissimilar to the phase- 

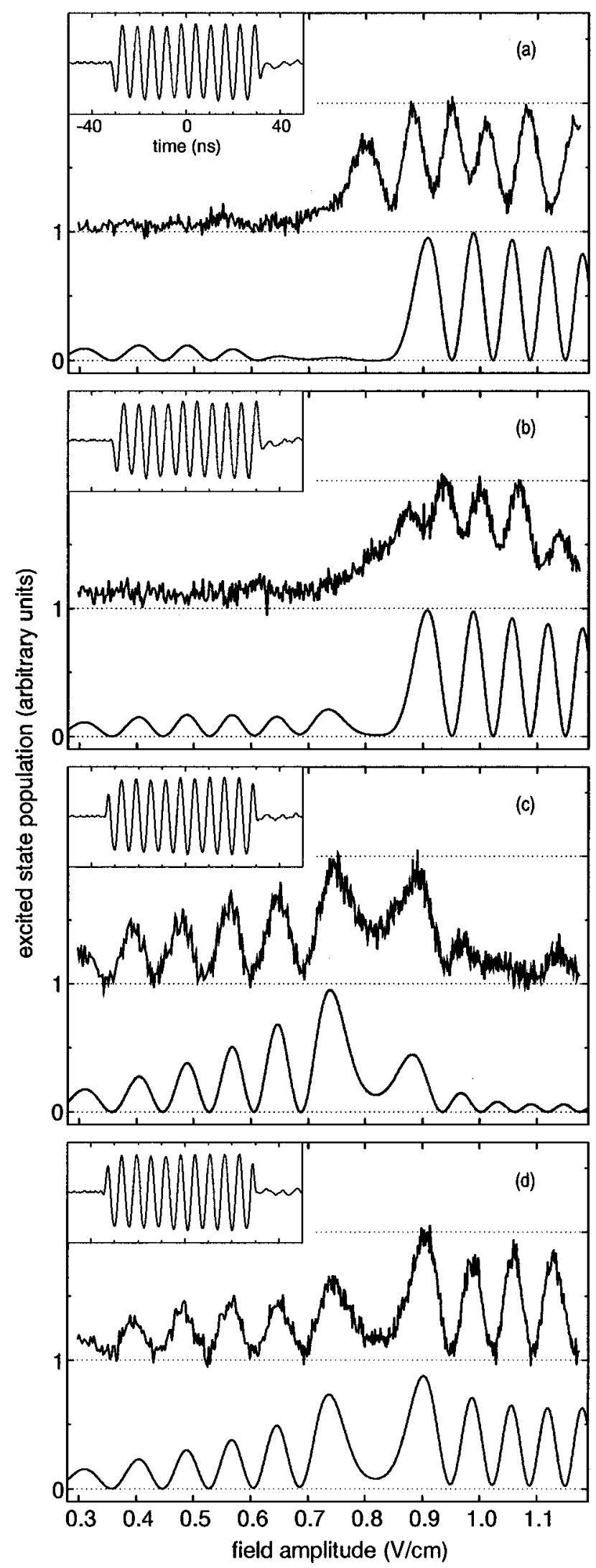

FIG. 8. Experimental result of amplitude scans with ten cycles at a frequency of $160 \mathrm{MHz}$ for several values of the rf pulse phase: (a) $\phi=90^{\circ}$, (b) $\phi=60^{\circ}$, (c) $\phi=0^{\circ}$, and (d) $\phi=-30^{\circ}$. The simulations shown immediately below the data were calculated using an rf pulse of 9.95 cycles and a $1.5-n s$ rise time.

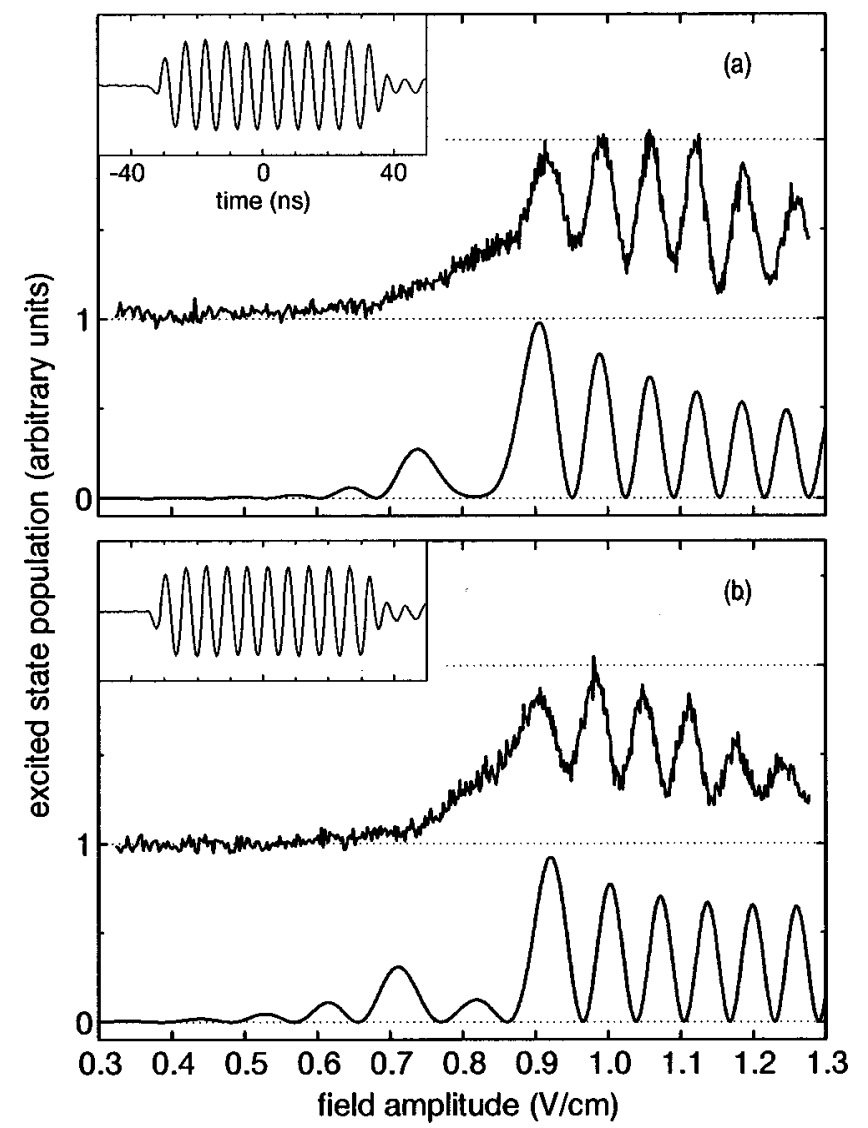

FIG. 9. Experimental result of rf pulse amplitude scans with ten-cycle pulses at $160 \mathrm{MHz}$ and a rise time of one cycle: (a) $\phi=90^{\circ}$ and (b) $\phi=0^{\circ}$. The simulations shown immediately below the data were calculated using an rf pulse of ten-cycle FWHM and a 6.3 -ns rise time.

and time-averaged results obtained with Floquet theory shown in Fig. 3(a).

The effects of an appreciable rise time can be understood in a simple way by examining the Fourier transform of an $N$ cycle pulse for both a sine and a cosine phase of the carrier. At a frequency $\omega_{0}$, both the sine and cosine transform into two sinc functions in the frequency domain, centered at $\omega= \pm \omega_{0}$. For the cosine, both central maxima are positive and add constructively on the high-frequency sides (i.e., for $\left.|\omega|>\omega_{0}\right)$ of the central extrema and destructively on the low-frequency sides $\left(|\omega|<\omega_{0}\right)$. For the sine, one central maximum of the sinc is positive and the other is negative, causing destructive interference on the high-frequency side of the central extrema $\left(|\omega|>\omega_{0}\right)$ and constructive interference on the low-frequency side. As the number of cycles is increased, more of the energy is concentrated in the carrier, apparently diminishing the importance of the frequency disparity between the two phases. However, the nonlinearity of the strongly driven two-level quantum system ensures that this small change in the frequency composition of the pulse can cause the observed difference in the response. In other words, the power in the difference of the spectra of the two phases is comparable to the power in a single cycle, which we know has a nontrivial affect on the system. The highfrequency components of the cosine are clearly caused by the very abrupt steps at the start and end of the pulse. The replacement of these steps with ramps of an appreciable rise 
time suppress the high-frequency components of the cosine pulse, whereas the sine pulse is not as dramatically affected. If we assume that the transitions in the low-amplitude region (i.e., with an amplitude less than the three-photon resonance) of the cosine rf pulse are in fact caused by the higherfrequency components driving a one-photon transition, then it is unsurprising that the imposition of an appreciable rise time that suppresses these components would also suppress the transitions.

While the preceding analysis is a good heuristic argument to understanding the transition probability when an $\mathrm{rf}$ pulse having an appreciable rise time is applied, it should be remembered that perhaps the only field that can be regarded by an atom as truly monochromatic is one that varies in time as $e^{i \omega t}$. This point of view is motivated by the fact that when a two-level atom is in a strong field, it is necessary to include the $e^{-i \omega t}$ counterrotating term, which will interfere with the rotating term. It may then be misleading to think that we can regard as separate the spectral components in a single rf pulse. We can then see that the phase- and time-averaged results of Shirley using an ostensibly monochromatic field were actually a particular average over the spectral components due to all of the phases being present. The use of the single cycle time propagator, which obtains transition probabilities for phase-dependent rf pulses, similarly folds in spectral components other than the carrier, but only those from a single phase. The significant consequence of this fact is that an rf pulse of sine phase does a reasonable job at modeling the response to an rf pulse of any phase with an appreciable rise time. The blind application of Shirley's time- and phase-averaged results to problems involving fast rise times can lead us astray because the process of phase averaging includes the high-frequency components in cosine phases, components that are actually suppressed in the real rf pulse because of its finite rise time.

The fact that an appreciable rise time can destroy the phase dependence then has some interesting consequences. When the envelope of the applied field is slowly varying, the use of Floquet theory as it has been reextended into the time domain is a good approximation of the two-level dynamics. In its most easily applied form, this method automatically performs a phase average, which as stated is not undesirable since the phase dependence nearly disappears. Where it is clear that this reextended Floquet theory must fail is in cases where the rf pulse envelope is not slowly varying and the phase is locked. In cases where the envelope is not slowly varying and the field has a fixed phase, it is necessary to integrate the Schrödinger equation numerically over the individual field cycles of the rf pulse. The propagator, however, is still a valuable numerical tool that can decrease the time needed to calculate transition probabilities over the parts of the field amplitude that are constant.

\section{CONCLUSIONS}

The single cycle time propagator is a powerful tool in the examination of any quantum system undergoing a timeperiodic interaction. It is related in a simple and transparent way to Floquet theory and contains all the information given by the Floquet Hamiltonian. While any particular single cycle propagator of a sinusoidal interaction depends on the phase with which it was evolved, its eigenvalues are independent of this phase and hence also contain phase- and time-averaged transition probabilities as given by Shirley. Nevertheless, the phase dependence is a strong point of the single cycle time propagator, as it is often more desirable to obtain such a result rather than a phase- and/or timeaveraged one. For example, in real pulses with appreciable rise and fall times (on the order of one period), the shapes of the resonances become phase independent but appear more like the fast-rise-time sine response rather than the phaseaveraged result that would be indicated by the Floquet picture for a constant field amplitude. These results are particularly important for ultrafast oscillations such as highintensity short pulse lasers, where rise times and pulse lengths are on the order of a few cycles [17].

\section{ACKNOWLEDGMENTS}

We would like to thank R. R. Jones for our many discussions on this study and Jim Carden at Intel Corp. for his help in obtaining and installing the Intel Fortran Reference compiler for use on an Intel donated Pentium Pro system. This work was supported by the Air Force Office of Scientific Research (AFOSR).
[1] J. H. Shirley, Phys. Rev. 138, B979 (1965).

[2] R. C. Stoneman, D. S. Thomson, and T. F. Gallagher, Phys. Rev. A 37, 1527 (1988).

[3] H. P. Breuer, K. Dietz, and M. Holthaus, Z. Phys. D 10, 13 (1988).

[4] M. C. Baruch and T. F. Gallagher, Phys. Rev. Lett. 68, 3515 (1992).

[5] J. G. Story, D. I. Duncan, and T. F. Gallagher, Phys. Rev. Lett. 70, 3012 (1993).

[6] R. B. Vrijen, J. H. Hoogenraad, H. G. Muller, and L. D. Noordam, Phys. Rev. Lett. 70, 3016 (1993).

[7] M. Gatzke, R. B. Watkins, and T. F. Gallagher, Phys. Rev. A 51, 4835 (1995).

[8] J. S. Melinger, Suketu R. Gandhi, A. Hariharan, J. X. Tull, and W. S. Warren, Phys. Rev. Lett. 68, 2000 (1992).
[9] B. W. Shore, K. Bergmann, A. Kuhn, S. Schiemann, J. Oreg, and J. H. Eberly, Phys. Rev. A 45, 5297 (1992).

[10] B. Broers, H. B. van Linden van den Heuvell, and L. D. Noordam, Phys. Rev. Lett. 69, 2062 (1992).

[11] W. R. Salzman, Phys. Rev. A 10, 461 (1974).

[12] J. V. Moloney and W. J. Meath, Mol. Phys. 30, 171 (1975).

[13] J. V. Moloney and W. J. Meath, Mol. Phys. 31, 1537 (1976).

[14] R. B. Watkins, W. M. Griffith, M. Gatzke, and T. F. Gallagher, Phys. Rev. Lett. 77, 2424 (1996).

[15] R. C. Stoneman and T. F. Gallagher, Phys. Rev. Lett. 55, 2567 (1985).

[16] R. C. Stoneman, G. Janik, and T. F. Gallagher, Phys. Rev. A 34, 2952 (1986).

[17] L. Xu, Ch. Spielmann, A. Poppe, T. Brabec, F. Krausz, and T. W. Hänsch, Opt. Lett. 21, 2008 (1996). 